\title{
Use of Open Software Tools for Data Offloading Techniques Analysis on Mobile Networks
}

\author{
José M. Koo, Juan P. Espino, Iván Armuelles, and Rubén Villarreal \\ Research Center for Information and Communication Technologies, \\ University of Panama, Republic of Panama \\ \{jkoo, jp.espino, ivan. armuelles, rvillarreal\} @citicup.org
}

\begin{abstract}
This research aims to highlight the benefits of using free software based tools for studying a LTE mobile network with realistic parameters. We will overload this LTE network and offload it through data offloading techniques such as small cells and WiFi offload. For this research, discreteevent open software network simulator ns3 will be implemented. Ns3 is a network simulator based on the programming language $\mathrm{C}++$, and has all the necessary libraries to simulate an LTE and WiFi network.
\end{abstract}

Keywords: Data Offloading, WiFi, LTE, small cells, ns3, OSS for research and education.

\section{Introduction}

In the past few years, demand on data transfer by mobile users has rapidly increased. Among many reasons, we can mention the rapid development of technologies which have enabled users to access higher transfer speed which in turn, enable them, for example, to make better quality video calls, download high quality videos, upload more files and such. On the other hand, social networks have boosted the total amount of data which traverse mobile networks. Because of this, mobile networks undergo a cicle of ever-increasing data rates to support the growing demand of users. This is why the capacity of mobile networks is slowly reaching its limit, and this simply leads to a degradation of the quality of customer service. According to [1], by $2018,15.9 \mathrm{~EB}\left(1 \mathrm{~EB}=10^{18}\right.$ bytes $)$ of monthly traffic is expected to be generated by mobile devices. There are a few techniques which allow mobile service providers to increase their mobile network capacity, nonetheless, the most efficient one is frequency reuse, which is posible by reducing the size of the cell.

Among those techniques, there is "data offloading" which seeks a way to offload those users who are in the mobile network and relocate them to another network, in this case, the Internet. This way, both, those who get to stay in the mobile network and those who have been relocated, will perceive an improvement in their quality of service.

Among the variants of data offloading techniques, we have small cells and WiFi offload. Small cells are low-powered base stations mainly designed for Small-OfficeHome-Office (SOHO) use. Small cells are compatible with current mobile communication technologies such as $4 \mathrm{G}$ and backward compatible with $2 \mathrm{G}$ and $3 \mathrm{G}$. 
On the other hand, we have WiFi offload, which is based on wireless Access point routers, through which the mobile user can connect and web-surf, make calls, among others. WiFi is considered a small cell as well, however, differ from them by the fact that WiFi operates in the unlicensed frequency spectrum.

It is necessary to evaluate the offload capacity that both techniques may offer, however, evaluation by hardware or non-open source software might be unviable, so the use of free open software tools is essential in our work.

\section{Objectives}

The objectives of our work are:

- Conduct a study with realistic parameters of a LTE network.

- Demonstrate that small cells and WiFi offload Access Points as data offloading techniques, reduce the load of a congested macrocell and compare the efficiency of small cells and WiFi Access Points and analyze their behaviour in tandem.

\section{$3 \quad$ Methodology}

To make this Project, the discrete-event open software network simulator ns3 will be used. Ns3 runs in a Linux environment and has both, LTE and WiFi libraries, which are necessary to simulate the proposed mobile network. Ns3 LTE libraries were developed by the Technical Telecommunication Center of Catalunya (CTTC) under the LENA Project. Our Project will consist of four (4) simulation scenarios which are summarized in Fig. 1.

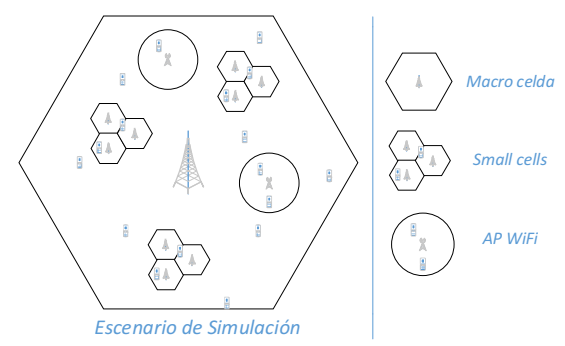

Fig. 1. Simulation scenarios and its components

\section{References}

[1] Cisco Visual Networking Index: Global Mobile Data Traffic Forecast Update, 2013-2018, Cisco

[2] SmallCellForum, http: / /www. smallcellforum.org

[3] ns3, http://www.nsnam.org 